\title{
Real-World Experience of
} Bevacizumab as First-Line Treatment for Ovarian Cancer: The GINECO ENCOURAGE Cohort of 468 French Patients

\section{OPEN ACCESS}

Edited by:

Nehad M. Ayoub,

Jordan University of Science and

Technology, Jordan

Reviewed by:

Gamal Eldein Fathy Abd-Ellatef Abd-

Elrahman,

National Research Centre, Egypt

Ajay Sharma,

University of Texas Health Science

Center at Houston, United States

*Correspondence:

Dominique Berton

dominique.berton@ico.unicancer.fr

Specialty section:

This article was submitted to Pharmacology of Anti-Cancer Drugs,

a section of the journal

Frontiers in Pharmacology

Received: 19 May 2021

Accepted: 17 August 2021

Published: 20 September 2021

Citation:

Berton D, Floquet $A$, Lescaut $W$, Baron G, Kaminsky M-C, Toussaint $P$, Largillier R, Savoye A-M, Alexandre Jô,

Delbaldo C, Malaurie E, Barletta $H$,

Bosacki C, Garnier-Tixidre C,

Follana $P$, Laharie-Mineur $H$,

Briac Levache C, Valenza B,

Dechartres A, Mollon-Grange $D$ and Selle Fé (2021) Real-World Experience

of Bevacizumab as First-Line

Treatment for Ovarian Cancer: The GINECO ENCOURAGE Cohort of 468

French Patients.

Front. Pharmacol. 12:711813.

doi: 10.3389/fphar.2021.711813
Dominique Berton $^{1 *}$, Anne Floquet ${ }^{2}$, Willy Lescaut ${ }^{3}$, Gabriel Baron ${ }^{4}$, Marie-Christine Kaminsky ${ }^{5}$, Philippe Toussaint ${ }^{6}$, Rémy Largillier $^{7}$, Aude-Marie Savoye ${ }^{8}$, Jérôme Alexandre ${ }^{9}$, Catherine Delbaldo ${ }^{10}$, Emmanuelle Malaurie ${ }^{11}$, Hugues Barletta ${ }^{12}$, Claire Bosacki ${ }^{13}$, Claire Garnier-Tixidre ${ }^{14}$, Philippe Follana ${ }^{15}$, Hortense Laharie-Mineur ${ }^{16}$, Charles Briac Levache ${ }^{17}$, Bruno Valenza ${ }^{18}$, Agnès Dechartres ${ }^{19}$, Delphine Mollon-Grange ${ }^{20}$ and Frédéric Selle ${ }^{10}$

${ }^{1}$ Institut de Cancérologie de l'Ouest, Saint-Herblain, France, ${ }^{2}$ Institut Bergonié, Bordeaux, France, ${ }^{3}$ Centre Hospitalier Princesse Grace, Monaco, Monaco, ${ }^{4}$ Assistance Publique - Hôpitaux de Paris Centre-Université de Paris, Hôpital Hôtel-Dieu, Centre d'Épidémiologie Clinique, Paris, France, ${ }^{5}$ Institut de Cancérologie de Lorraine, Vandœuuvre-lès-Nancy, France, ${ }^{6}$ Centre Léon Bérard, Lyon, France, ${ }^{7}$ Centre Azuréen de Cancérologie, Mougins, France, ${ }^{8}$ Institut Jean Godinot, Reims, France, ${ }^{9}$ Université de Paris, Hôpital Cochin, Paris, France, ${ }^{10}$ Groupe Hospitalier Diaconesses Croix Saint Simon, Paris, France, ${ }^{11}$ Centre Hospitalier Intercommunal de Créteil, Créteil, France, ${ }^{12}$ Centre Mistral, Guilherand-Granges, France, ${ }^{13}$ Institut de Cancérologie de la Loire, Saint-Priest-en-Jarez, France, ${ }^{14}$ Groupe Hospitalier Mutualiste de Grenoble, Grenoble, France, ${ }^{15}$ Centre Antoine Lacassagne, Nice, France, ${ }^{16}$ Clinique Tivoli, Bordeaux, France, ${ }^{17}$ Clinique Francheville, Périgueux, France, ${ }^{18} \mathrm{Centre} \mathrm{Hospitalier} \mathrm{Intercommunal}$ de Fréjus, Saint-Raphaël, France, ${ }^{19}$ Sorbonne Université, Assistance Publique - Hôpitaux de Paris, Hôpitaux Universitaires Pitié Salpêtrière - Charles Foix, Paris, France, ${ }^{20}$ Centre Hospitalier Intercommunal de Cornouaille, Quimper, France

Introduction: Bevacizumab-containing therapy is considered a standard-of-care frontline option for stage IIIB-IV ovarian cancer based on results of randomized phase 3 trials. The multicenter non-interventional ENCOURAGE prospective cohort study assessed treatment administration and outcomes in the French real-world setting.

Patients and Methods: Eligible patients were aged $\geq 18$ years with planned bevacizumab-containing therapy for newly diagnosed ovarian cancer. The primary objective was to assess the safety profile of front-line bevacizumab in routine clinical practice; secondary objectives were to describe patient characteristics, indications/ contraindications for bevacizumab, treatment regimens and co-medications, follow-up and monitoring, progression-free survival, and treatment at recurrence. In this noninterventional study, treatment was administered as chosen by the investigator and participation in the trial had no influence on the management of the disease.

Results: Of 1,290 patients screened between April 2013 and February 2015, 468 were eligible. Most patients (86\%) received bevacizumab $15 \mathrm{mg} / \mathrm{kg}$ every 3 weeks or equivalent, typically with carboplatin (99\%) and paclitaxel (98\%). The median duration of bevacizumab

Abbreviations: CI, confidence interval; FIGO, International Federation of Gynecology and Obstetrics; GINECO, Groupe d'Investigateurs Nationaux pour l'Etude des Cancers de l'Ovaire et du sein; INCa, French National Cancer Institute; OS, overall survival; PFS, progression-free survival; PARP, polyADP ribose polymerase. 
was 12.2 (range 0-28, interquartile range 6.9-14.9) months; 8\% of patients discontinued bevacizumab because of toxicity. The most common adverse events were hypertension (38\% of patients), fatigue (35\%), and bleeding (32\%). There were no treatment-related deaths. Most physicians (90\%) reported blood pressure measurement immediately before each bevacizumab infusion and almost all (97\%) reported monitoring for proteinuria before each bevacizumab infusion. Median progression-free survival was $17.4(95 \% \mathrm{Cl}$, 16.4-19.1) months. The 3-year overall survival rate was $62 \%(95 \% \mathrm{Cl}, 58-67 \%)$. The most commonly administered chemotherapies at recurrence were carboplatin and pegylated liposomal doxorubicin.

Discussion: Clinical outcomes and tolerability with bevacizumab in this real-life setting are consistent with randomized trial results, notwithstanding differences in the treated patient population and treatment schedule.

Clinical Trial Registration: ClinicalTrials.gov, Identifier NCT01832415.

Keywords: bevacizumab, ovarian cancer, routine clinical practice, monitoring, progression-free survival

\section{INTRODUCTION}

In two randomized phase 3 trials (GOG-0218 and ICON7), combining bevacizumab with a carboplatin-paclitaxel doublet for newly diagnosed ovarian cancer significantly improved progression-free survival (Burger et al., 2011; Perren et al., 2011), leading to European Medicine's Agency (EMA) regulatory approval for stage IIIB, IIIC, and IV ovarian cancer. More recently, bevacizumab-containing therapy was approved by the US Food and Drug Administration for stage III or IV ovarian cancer following initial surgical resection (Avastin Prescribing Information, 2020).

In the GOG-0218 trial, bevacizumab was associated with a significant increase in grade $\geq 2$ hypertension versus chemotherapy alone (Burger et al., 2011). However, hypertension was generally manageable and rarely required treatment discontinuation. Bevacizumab was also associated with increased grade $\geq 3$ proteinuria, which appears to be cumulative, and grade $\geq 2$ gastrointestinal events, which were nevertheless relatively infrequent. Most adverse events occurred during the combination chemotherapy phase rather than during single-agent bevacizumab maintenance therapy. Similar patterns were seen in the ICON7 trial using a lower dose and shorter treatment duration of bevacizumab (Perren et al., 2011).

In France, more than 5,000 new cases of ovarian cancer are diagnosed and almost 3,500 women die from ovarian cancer each year (Institut National du Cancer (INCa), 2021). Bevacizumab was rapidly adopted into routine clinical practice in France and according to guidelines endorsed by the French National Cancer Institute (INCa), adding bevacizumab to chemotherapy should be proposed to patients with International Federation of Gynecology and Obstetrics (FIGO) stage III and IV disease, particularly in those with a poor prognosis (stage IV, residual disease after surgery, or inoperable disease) (de la Motte Rouge et al., 2019). The ENCOURAGE study aimed to collect information on treatment administration and clinical outcomes in the realworld setting from a patient population more representative of routine clinical practice than selected populations eligible for phase 3 trials. We report final results from ENCOURAGE.

\section{MATERIALS AND METHODS}

ENCOURAGE (NCT01832415) was a multicenter noninterventional single-arm prospective French cohort study led by the Groupe d'Investigateurs Nationaux pour l'Etude des Cancers de l'Ovaire et du sein (GINECO). The study was conducted in accordance with the ethical principles of the Helsinki agreement and all subsequent amendments, conformed to French legal requirements, and was approved by the Comité Consultatif sur le Traitement de l'Information en Matière de Recherche dans le Domaine de la Santé and the Commission Nationale de l'Informatique et des Libertés. All patients consented verbally to participate and the treating physician signed a form of non-objection, noting that the patient had consented to have their clinical data recorded in the framework of this non-interventional study.

The primary objective was to assess the safety profile of frontline bevacizumab therapy for ovarian cancer in routine clinical practice, focusing particularly on cardiovascular (hypertension, thromboembolism), renal (proteinuria), hemorrhagic (bleeding), gastrointestinal (gastrointestinal perforation, fistula, abscess), and post-operative effects. Secondary objectives included describing the characteristics of patients treated in routine practice, evaluating bevacizumab indications and contraindications in presenting patients, describing treatment regimens (dose, duration, and chemotherapy partner) and co-medications (particularly antihypertensives) used in routine practice, evaluating patient follow-up and monitoring (type and frequency of blood pressure monitoring, proteinuria assessment), evaluating progression-free survival (PFS) and overall survival (OS), and evaluating subsequent chemotherapy at recurrence.

Women aged $\geq 18$ years, with a diagnosis of ovarian, fallopian tube, or primary peritoneal cancer planned for first-line 
bevacizumab-containing therapy were eligible. Participation in another clinical trial while participating in ENCOURAGE was prohibited.

The study was conducted in centers representative of patients presenting with ovarian cancer in routine oncology practice in France (private practice, non-academic hospitals, academic hospitals). Patients were followed for 36 months according to each participating center's standard practice. Clinical data were recorded in an electronic case report form at baseline and at 6, 12, 18 , and 36 months after initiating bevacizumab.

Data were reported using descriptive statistics [frequency (\%) for categorical data and median (range or interquartile range) for quantitative data]. OS and PFS with 95\% confidence intervals (CIs) and median survival with 95\% CIs were estimated using Kaplan-Meier methodology. Data were analyzed using SAS Version 9.4 (SAS Institute) and R Version 3.5.1.

\section{RESULTS}

Between April 2013 and February 2015, 1,290 patients at 103 participating centers involving 205 investigators were screened. Of these, 790 patients were not enrolled for participation in this non-interventional study because of: age $(n=70)$; medical contraindication $(n=41)$; FIGO stage I-IIIA $(n=70)$; FIGO stage IIIB-IV with no residual disease $(n=23)$; planned interval surgery $(n=32)$; or other reason $(n=593)$ (more than one reason possible) (Figure 1). The most commonly reported contraindications were a history of venous thrombosis $(n=$ 20 ) or a history of fistula, perforation, or abscess $(n=9)$. Of the 500 patients ultimately enrolled, 468 were included in the analysis and 32 were not evaluable (Figure 1).

Table 1 shows baseline characteristics. Of the 468 analyzed patients, $164(35 \%)$ were treated in private practice, $131(28 \%)$ in non-academic hospitals, 99 (21\%) in anticancer centers, and 74 (16\%) in academic hospitals. Most investigators (141 of 187 who enrolled analyzed patients; $75 \%$ ) had been involved in clinical trials within the preceding 3 years and 117 (63\%) participated in gynecology societies.

Most patients (405 of 468 ; 87\%) received bevacizumab at a dose of $15 \mathrm{mg} / \mathrm{kg}$ every 3 weeks or equivalent. Of the remainder, $38(8 \%)$ received bevacizumab at a dose of $7.5 \mathrm{mg} / \mathrm{kg}$ every 3 weeks (as used in ICON7) and $25(5 \%)$ received another schedule. Bevacizumab was combined with carboplatin in 464 patients (99\%) and paclitaxel in 458 patients (98\%) [other chemotherapy partners included gemcitabine in four patients $(0.9 \%)$, oxaliplatin and docetaxel each in two patients $(0.4 \%)$, and cyclophosphamide in one patient $(0.2 \%)]$. By the data cut-off date, all but four patients had discontinued bevacizumab. The median interval between initiating chemotherapy and first bevacizumab dose was 77.5 (range 0-494, interquartile range 21-171) days. The median number of bevacizumab cycles was 18 (range 1-53, interquartile range 10-21), corresponding to a median duration of 12.2 (range $0-28$, interquartile range 6.9-14.9) months. Bevacizumab was continued beyond the planned 15 months in 106 patients (23\%); reasons for prolonging treatment were not collected. Among the 463 patients who had discontinued bevacizumab at the data cutoff, bevacizumab treatment was discontinued because of disease progression in 169 patients (37\%) and because of toxicity in $36(8 \%)$.

The most common adverse events were hypertension (any grade in 177 patients; $38 \%$ ), fatigue (166 patients; $35 \%$ ), bleeding [148 patients, 32\%; predominantly epistaxis (152 episodes) and gum bleeding (48 episodes)], pain (96 patients, 21\%), headache (63 patients, 13\%), and arthralgia (59 patients, 13\%) (Figure 2). Six patients (1\%) developed posterior reversible leukoencephalopathy syndrome, which led to hospitalization in one patient. Gastrointestinal perforation, fistula, and arterial events were infrequent (Figure 2). Proteinuria occurred in 25 patients (5\%). There were no cases of congestive heart failure and no treatment-related deaths. Serious adverse events were reported in 17 patients (4\%; 18 events), of which one event was grade 4,13 were grade 3 , three grade 2 , and one of unknown grade.

Most physicians (168 of 187; 90\%) reported blood pressure measurement immediately before each bevacizumab infusion; 14 (7\%) reported blood pressure measurement several times between each infusion; and five (3\%) reported less frequent blood pressure measurement. Table 2 provides details of hypertension detection and management. Hypertension was most frequently detected by an oncologist (58\%), and $68 \%$ of episodes required initiation or modification of antihypertensive therapy. Almost all physicians (181; 97\%) reported monitoring for proteinuria before each bevacizumab infusion, typically using a urine dipstick. 24-h protein monitoring was requested following a urine dipstick result of $2+$ by 103 clinicians (55\%) and of $3+$ in $66(35 \%)$ [1+ in $7(4 \%)$, missing in $11(6 \%)]$.

Overall, 165 patients (35\%) underwent 234 surgical procedures after initiating bevacizumab. Surgery comprised 91 tumor resections, 45 abdominopelvic procedures to treat complications, 23 central line procedures, 13 dental procedures, and "other" procedures in the remaining 62 cases (30 of which were considered minor, such as ascites puncture, urinary catheter ablation, colonoscopy/gastroscopy). Most (116 patients, 25\%) underwent only one surgical procedure but 35 patients had two procedures, nine had three procedures, four had four procedures, and one had five procedures. The median interval between last bevacizumab dose and surgery was 2.1 months (range $0-30$ months, interquartile range 1.2-9.7). Secondary effects or complications were observed within 30 days following surgery in $34(15 \%)$ of the 234 procedures, most commonly mild-to-moderate wound-healing complications (eight procedures; 3\%), moderate-to-severe infection (four procedures; $2 \%$ ), or moderate bleeding (four procedures; $2 \%$ ). There were three cases of intestinal perforation or fistula within 30 days of surgery (one mild, two severe).

At the data cut-off (August 23, 2018), the median follow-up duration was 35.2 months (range 1.8-44.0; interquartile range 25.4-36.3). At this date, 273 patients $(58 \%)$ had completed follow-up as planned, $172(37 \%)$ had died, 18 (4\%) were lost to follow-up before completing the protocol-required 36-months follow-up, three (1\%) were being followed in another hospital, and two $(<1 \%)$ had discontinued because of technical/ administrative reasons. At the data cut-off, 114 patients (24\%) 


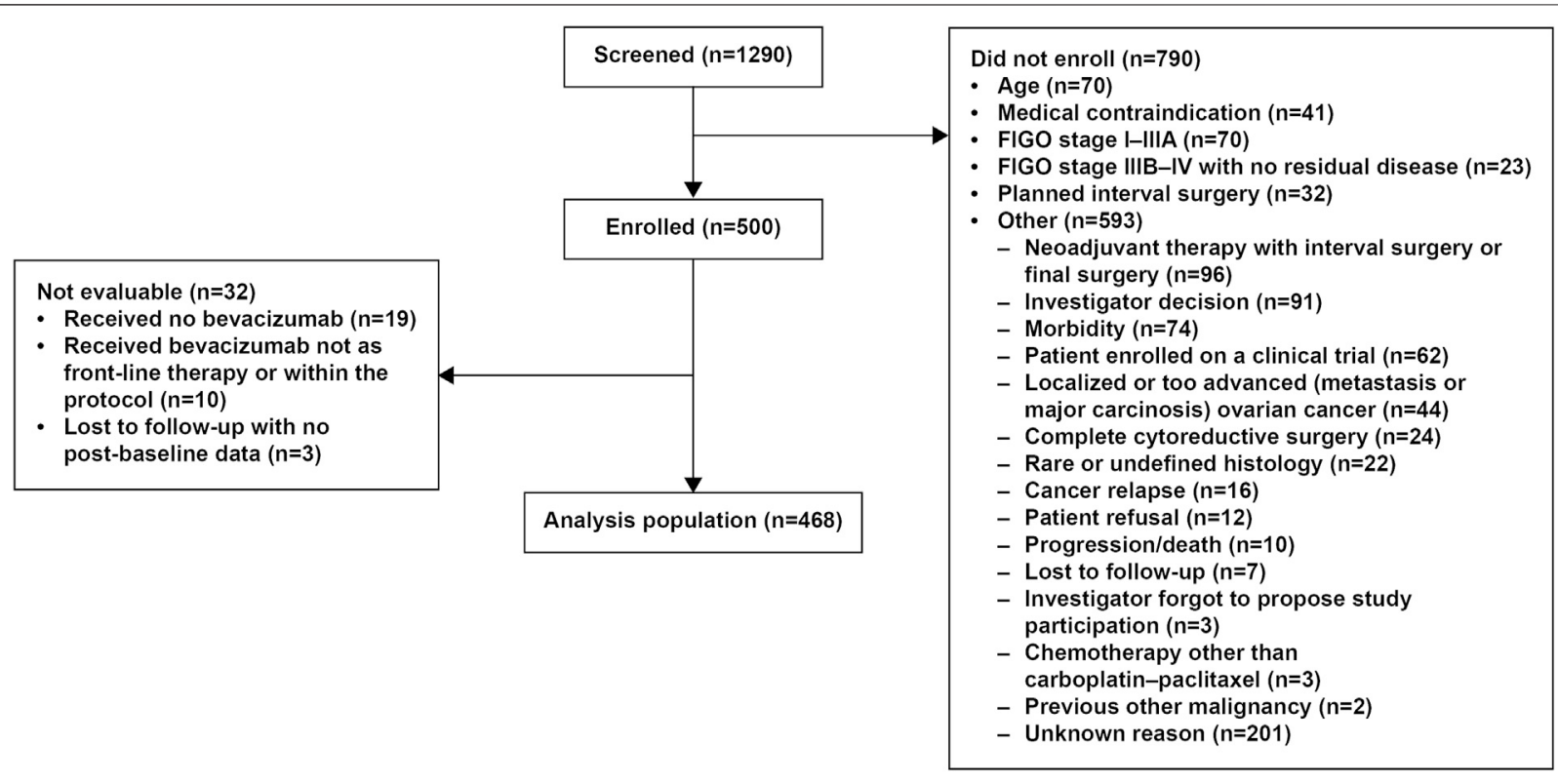

FIGURE 1 | Patient flow. More than one reason for not enrolling is possible. A total of 667 "other" reasons were reported in 593 patients.

were alive without progression, 349 (75\%) had experienced disease progression, and five (1\%) had died without evidence of progression. Median PFS was 17.4 (95\% CI 16.4-19.1) months (Figure 3A). The 1-year PFS rate was 68\% (95\% CI, 64-73\%) and the 3-year PFS rate was 25\% (95\% CI, 21-29\%). Overall, 172 patients (37\%) had died at the date of study completion. All but 11 deaths were attributable to disease progression. The 3-year OS rate was 62\% (95\% CI, 58-67\%) (Figure 3B).

Information on subsequent therapy was available for 327 (94\%) of the 349 patients with recorded disease recurrence. The most commonly administered chemotherapy (alone or in combination) at recurrence was carboplatin (219 of 327 patients; $67 \%)$, followed by pegylated liposomal doxorubicin (181;55\%). Forty-four patients (13\%) received bevacizumab therapy and 19 $(6 \%)$ received olaparib.

\section{DISCUSSION}

In French routine practice, front-line bevacizumab is usually administered according to the EMA label. The vast majority of patients received bevacizumab $15 \mathrm{mg} / \mathrm{kg}$ every 3 weeks combined with a carboplatin-paclitaxel doublet. Clinical outcomes and tolerability in this real-life setting are consistent with reports from randomized trials, notwithstanding differences in the treated patient population and treatment schedule.

Since initiating this study, results have been published from the single-arm phase 3B ROSiA study in more than 1,000 patients from 35 countries (Oza et al., 2017). ROSiA adopted a pragmatic, real-world approach, with clinicians choosing their preferred schedule of paclitaxel (weekly or every 3 weeks) and bevacizumab (although most chose $15 \mathrm{mg} / \mathrm{kg}$ every 3 weeks). Importantly, bevacizumab could be continued for up to
24 months, or even longer if patients were still deriving clinical benefit. Until recently, the optimal bevacizumab treatment duration had been an unanswered question in the gynecologic oncology community ever since the GOG-0218 and ICON7 trials were first presented (Marth et al., 2017; Musella et al., 2017). Results from the single-arm ROSiA study hinted toward improved outcomes with longer treatment duration, but had inherent bias because perceived benefit was not assessed by imaging. In the ENCOURAGE study, most patients received treatment until disease progression and the median treatment duration was 18 cycles (interquartile range 10-21). Almost one fourth of patients received bevacizumab for longer than the planned 15 months, yet median PFS was consistent with trials evaluating 15 months of bevacizumab. Recently, definitive results on bevacizumab duration were reported from the randomized phase 3 AGOOVAR17/BOOST trial (NCT01462890) comparing 15 versus 30 months of bevacizumab. Longer bevacizumab treatment duration did not improve PFS or OS, and therefore 15 months of bevacizumab remains the standard duration (Pfisterer et al., 2021).

One of the main objectives of the ENCOURAGE study was to assess tolerability, particularly the incidence and management of selected adverse events typically associated with bevacizumab-containing therapy. The $38 \%$ incidence of anygrade hypertension appears to be higher than the $26 \%$ incidence reported in ICON7 with bevacizumab $7.5 \mathrm{mg} / \mathrm{kg}$ every 3 weeks for 12 months (Perren et al., 2011) and marginally higher than the $32 \%$ incidence in GOG-0218 with bevacizumab $15 \mathrm{mg} / \mathrm{kg}$ every 3 weeks for 15 months (Avastin Prescribing Information, 2020). However, given the focus on hypertension in the ENCOURAGE case report form, increased recognition of this side effect, and the requirement to record the frequency of 
TABLE 1 | Baseline characteristics of analyzed patients $(N=468)$.

ECOG performance status

0

1

2

3

Not done

Pre-existing conditions at study entry ${ }^{a}$

Arterial hypertension

Type 2 diabetes

$\mathrm{BMI}>30 \mathrm{~kg} / \mathrm{m}^{2}$

Ongoing treatment at study entry ${ }^{a}$

Antihypertensive therapy

Statin

Anticoagulant therapy

FIGO stage at diagnosis

I

II

IIIA

IIIB-IV

Unknown

Histology

Serous

Endometrioid

Clear cell

Mucinous

Other

Primary surgery including laparoscopy for staging

Complete debulking

Interval debulking surgery before starting bevacizumab

$155(33)$

$218(47)$

$19(4)$

$1(<1)$

75 (16)

$148(32)$

$130(28)$

$20(4)$

$30(6)$

177 (38)

$124(26)$

$41(9)$

46 (10)

$6(1)$

5 (1)

5 (1)

418 (89)

$34(7)$

Complete interval debulking

387 (83)

36 (8)

7 (1)

7 (1)

$31(7)$

$427(91)$

$125(27)$

220 (47)

156/464 (34)

BRCA mutation status

Mutant

$B R C A 1^{a}$

BRCA2 ${ }^{\mathrm{a}}$

38 (8)

24 (5)

16 (3)

Wild type

109 (23)

Unknown

321 (69)

Baseline urine dipstick result

( $n=341)$

292 (62)

Trace

$25(5)$

$1+$

$2+$

24-h protein measurement

Median (range) [interquartile range], g/24 h $(n=13)$

$18(4)$

$6(1)$

$13 / 467$ (3)

$0.1(0-1)[0.1-0.2]$

${ }^{a}$ More than one answer possible.

BMI, body mass index; ECOG, Eastern Cooperative Oncology Group; FIGO, International Federation of Gynecology and Obstetrics.

monitoring, increased vigilance for hypertensive events may have contributed to greater awareness and reporting. Additionally, the population was less selected in ENCOURAGE than in randomized phase 3 trials. The incidence of all-grade hypertension in ENCOURAGE appears to be lower than the $55 \%$ incidence reported in the single-arm ROSiA study, in which bevacizumab was administered for up to 36 months typically at $15 \mathrm{mg} / \mathrm{kg}$ every 3 weeks. As noted by the ROSiA investigators, hypertension may occur in later cycles and therefore the treatment duration in ENCOURAGE (longer than in ICON7, shorter than in ROSiA) may contribute to the hypertension incidence observed in ENCOURAGE (higher than in ICON7, lower than in ROSiA). Differences in the grading scale for assessing hypertension may also contribute (Oza et al., 2017; Plummer et al., 2019).

Another well-documented side effect of bevacizumabcontaining therapy is epistaxis. In ENCOURAGE, the vast majority of bleeding events were epistaxis. The $32 \%$ incidence of bleeding is similar to the $39 \%$ incidence reported in ICON7 (Perren et al., 2011). Few trials report low-grade bleeding as this is generally considered a manageable event requiring minimal or no medical intervention (Miles et al., 2010). Patients may experience bleeding gums, blood-stained mucus after nose blowing, or epistaxis requiring only basic first aid (Miles et al., 2010).

Gastrointestinal perforation occurred in only one patient (0.5\%), no higher than in the ICON7 trial (Perren et al., 2011) 


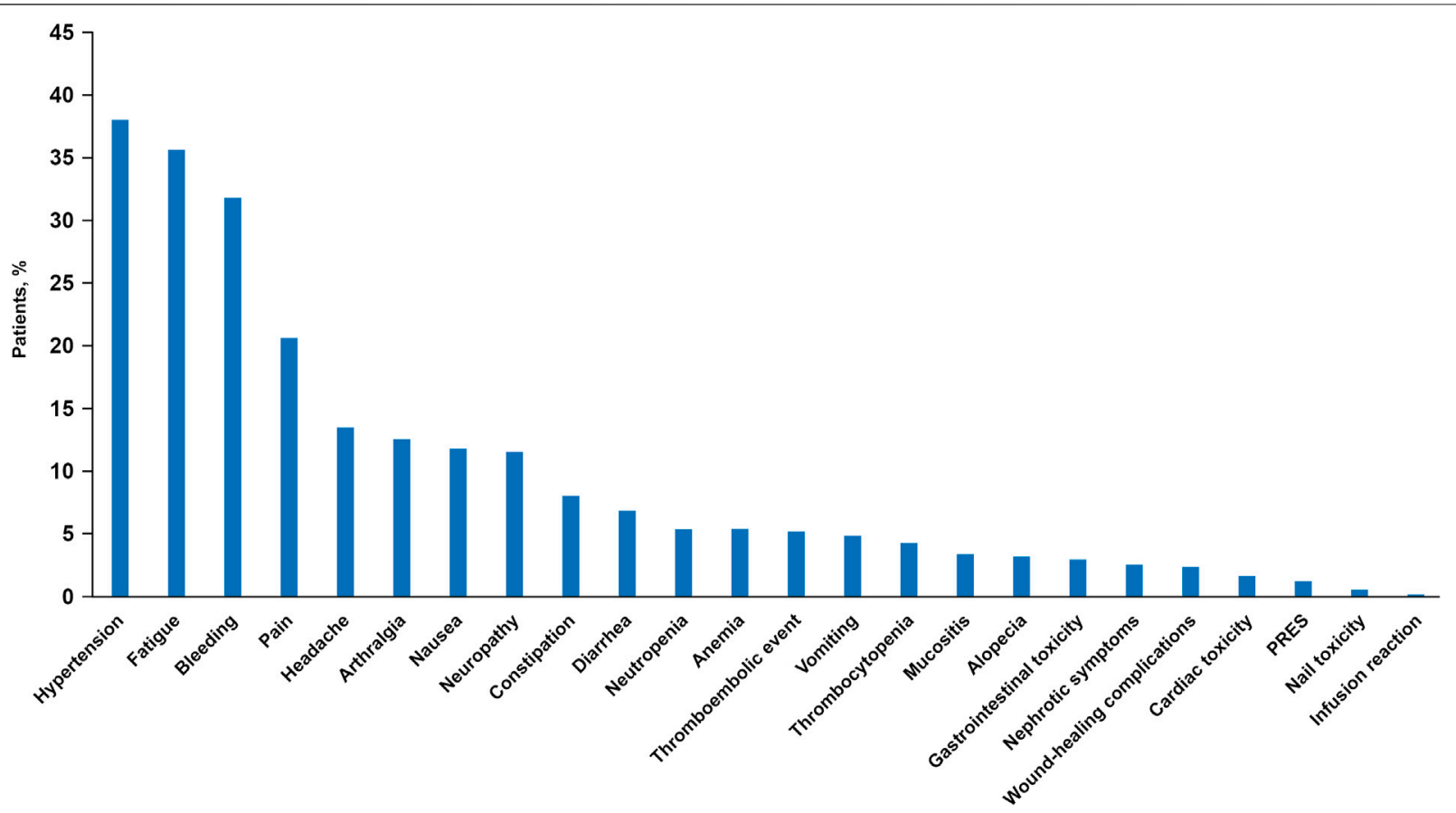

FIGURE 2 | Percentage (in descending order) of patients experiencing adverse events (all grades). Verbatim terms recorded under target adverse event categories were as follows: thromboembolic events: phlebitis $(n=8)$, pulmonary embolism $(n=6)$, cerebrovascular accident $(n=1)$, other $(n=12)$. Gastrointestinal toxicity: subileus $(n=2)$, gastrointestinal perforation, constipation, abdominal distention, upper abdominal pain, dyspepsia, fistula, enterocutaneous fistula, perineal fistula, umbilical hernia, intestinal occlusion, wound evisceration/stomal complication/fistula/dehiscence/urinoma, gastrointestinal disorders (each $n=1$ ). Cardiac toxicity: hypertension $(n=2)$, palpitations $(n=2)$, pulmonary artery hypertension, aortic insufficiency/palpitations/supraventricular extrasystole, acute coronary syndrome, tachycardia, poorly defined (each $n=1$ ). PRES, posterior reversible encephalopathy syndrome.

TABLE 2 | Description of hypertensive episodes in patients receiving bevacizumab therapy.

\section{Hypertension diagnosis and management}

Healthcare professional detecting hypertensive episode ${ }^{a}$

Oncologist

Nurse

General practitioner

Cardiologist

Other

Consultation with cardiologist

Median maximal blood pressure (range) [interquartile range], $\mathrm{mmHg}$

Systolic $(n=229)$

Diastolic $(n=216)$

Antihypertensive therapy initiated or modified ${ }^{b}$

First therapy initiated

Additional drug administered

Class of antihypertensive changed

Dose increased

Type of antihypertensive drug(s) ${ }^{\mathrm{b}}$

Calcium channel blocker

Beta blocker

Angiotensin-converting enzyme inhibitor

Angiotensin receptor blocker

Thiazide diuretic

Other $^{\mathrm{C}}$

No. of episodes (\%)

$149(58)$

$72(28)$

25 (10)

15 (6)

$27(11)$

90/257 episodes (35)

165 (20-220) [154-180]

90 (55-193) [81.5-100]

$176 / 257$ (68)

98 (38)

46 (18)

37 (14)

29 (11)

114 (44)

64 (25)

64 (25)

60 (23)

35 (14)

9 (4)

${ }^{a}$ A single hypertensive episode could be detected by more than one healthcare professional.

${ }^{b}$ Patient may receive more than one anti-hypertensive drug.

${ }^{c}$ Alpha-adrenoreceptor antagonist $(n=6)$, combination of beta blocker and thiazide diuretic $(n=1)$, loop diuretic $(n=1)$, other peripheral vasodilator $(n=1)$.

257 hypertensive episodes (any grade) reported among 177 of the 468 analyzed patients. 


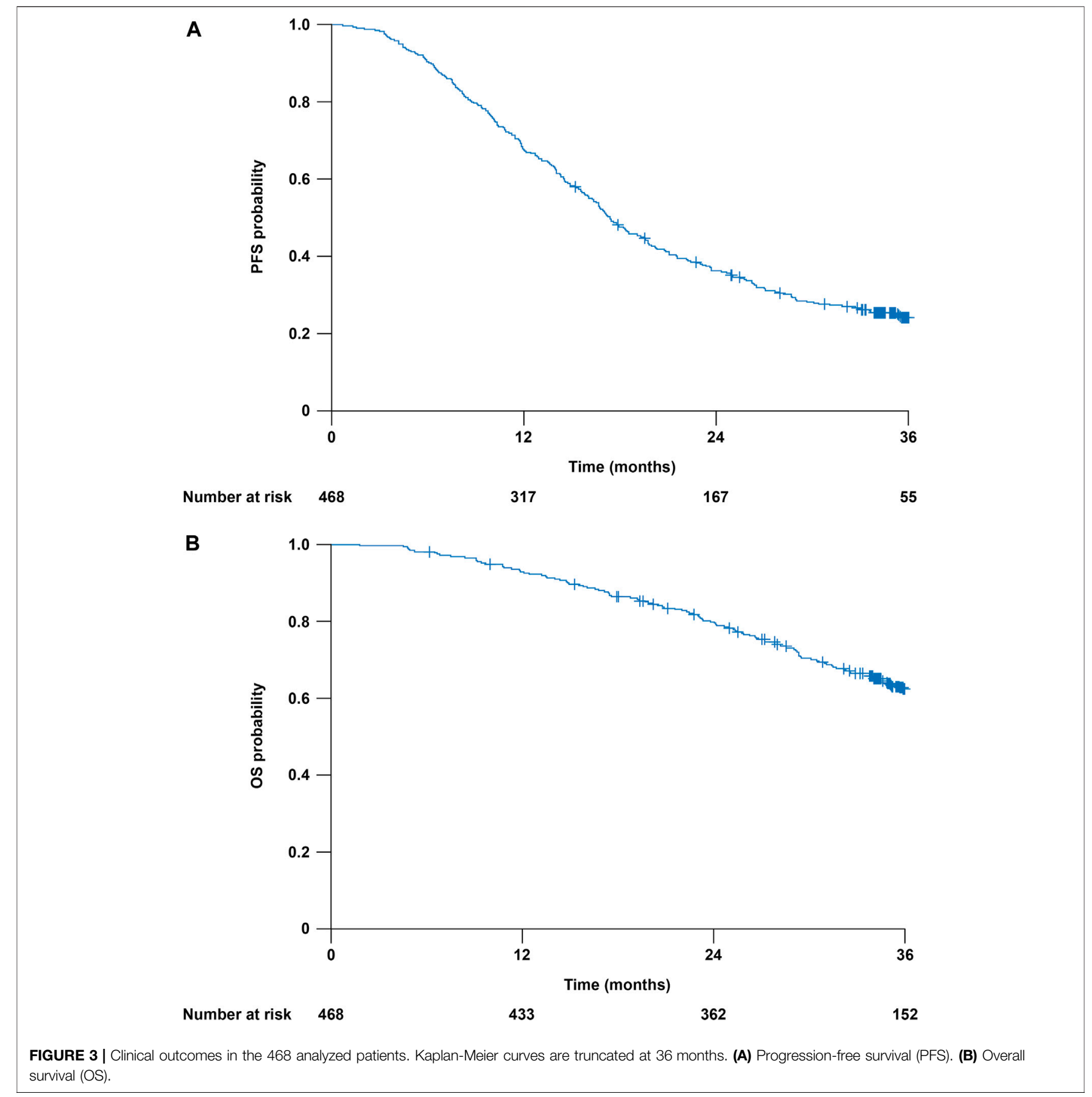

or ROSiA (Oza et al., 2017), and perhaps reflecting increased familiarity and caution surrounding this side effect. The 5\% incidence of proteinuria (any grade) in ENCOURAGE is within the range reported in ICON7 (5\% any grade with a shorter duration of bevacizumab) and ROSiA (4\% grade $\geq 3$ with a longer duration of bevacizumab), although in the UK OSCAR study proteinuria was reported at any grade in $20 \%$ of patients and at grade $\geq 3$ in $1 \%$ (Hall et al., 2020). All such cross-trial comparisons should be interpreted with considerable caution given differences in study design, patient selection, treatment administration, and adverse event monitoring and follow-up.

A potential weakness of the study is the less rigorous monitoring and data collection and more heterogeneous patient population and clinical assessment typical of an observational study, such as ENCOURAGE, versus a doubleblind, placebo-controlled randomized phase 3 trial. However, this is also the strength and objective of the study: to explore whether 
outcomes in highly selected populations can be replicated in the real-world setting in patients with more comorbidities and frailties that may prevent them enrolling into randomized clinical trials. Similar studies reporting outcomes in routine clinical practice have been reported from the UK, Germany, and Japan (Komiyama et al., 2019; Hall et al., 2020; Sehouli et al., 2020). As with ENCOURAGE, these studies showed a safety profile consistent with phase 3 reports, with no new safety signals. All-grade hypertension was reported in $32 \%$ of patients in OSCAR, $23 \%$ in JGOG3022, and $17 \%$ in OTILIA. A limitation in such comparisons is that reporting of adverse events was in accordance with local practice, and therefore subject to reporting bias. A strength of these country-specific studies compared with global studies such as ROSiA is the more homogenous population, representing healthcare systems in a single country and thus providing more relevant information on expected outcomes for oncologists in those countries to discuss with their patients. For example, in the United Kingdom OSCAR study, only $21 \%$ of patients underwent primary debulking surgery and $43 \%$ received no surgery (Hall et al., 2020), whereas in the French population treated in ENCOURAGE, 91\% underwent primary surgery and $47 \%$ had interval debulking surgery, resulting in complete resection in $27 \%$ and $33 \%$ of patients, respectively.

Median PFS of 17.4 months in ENCOURAGE is within the range reported in previous prospective trials evaluating bevacizumab therapy for up to 15 months [GOG-0218, ICON7, OTILIA, OSCAR, JGOG3022: 15.4-19.4 months (Burger et al., 2011; Perren et al., 2011; Komiyama et al., 2019; Avastin Prescribing Information, 2020; Hall et al., 2020; Sehouli et al., 2020)] but shorter than the median PFS of 25.5 months reported in the ROSiA study (Oza et al., 2017). Most recently, results from the PAOLA-1 randomized phase 3 trial showed median PFS of 16.6 months with front-line bevacizumab and chemotherapy (consistent with findings in our study), but this was increased to 22.1 months with the addition of maintenance olaparib therapy (Ray-Coquard et al., 2019). Enrollment to the ENCOURAGE study was completed before polyADP ribose polymerase (PARP) inhibitors became standard-of-care therapy in the recurrent setting and before data were available in the front-line setting. One may assume that in France, many of the population treated in ENCOURAGE, particularly those with $B R C A$-mutated tumors, would now be considered for maintenance olaparib after completing chemotherapy. At recurrence, relatively few patients received bevacizumab retreatment; based on results from the MITO16B-MaNGO OV2B-ENGOT OV17 randomized phase 3 trial (Pignata et al., 2021), bevacizumab re-treatment after front-line bevacizumabcontaining therapy seems a reasonable option.

Findings from ENCOURAGE suggest that continued education about the importance of blood pressure monitoring and prompt management of low-grade hypertension remains important in patients receiving bevacizumab. Recently published consensus guidelines developed by cardiologists, medical oncologists, a general practitioner, and specialist oncology nurses, all with experience of treating patients with bevacizumab and/or hypertension, recommend blood pressure measurement in all patients before each bevacizumab infusion and provide clear recommendations for hypertension management (Plummer et al., 2019). They also recommend close communication between a patient's oncologist and general practitioner and clear specific instructions about target blood pressure and management.

In summary, these results from patients with newly diagnosed ovarian cancer treated with bevacizumab-containing therapy in a real-life setting were relatively consistent with findings from the randomized phase 3 GOG-0218 and ICON7 trials (Burger et al., 2011; Perren et al., 2011). Continued education on blood pressure monitoring and management of hypertension remains important, particularly as the use of bevacizumab with PARP inhibition becomes standard of care.

\section{DATA AVAILABILITY STATEMENT}

The datasets presented in this article are not readily available because currently no mechanism is in place to allow sharing of individual deidentified participant data. Requests to access the datasets should be directed to ARCAGY-GINECO (bvotan@ arcagy.org) and will be considered on a case-by-case basis.

\section{ETHICS STATEMENT}

The study was reviewed and approved by the Comite Consultatif sur le Traitement de l'Information en Matière de Recherche dans le Domaine de la Santé and the Commission Nationale de l'Informatique et des Libertés. Written informed consent for participation was not required for this study in accordance with the national legislation and the institutional requirements.

\section{AUTHOR CONTRIBUTIONS}

Conceptualization and methodology: $\mathrm{DB}$ and AD. Statistical analysis: GB. Patient enrollment: DB, AF, WL, M-CK, PT, RL, A-MS, JA, CD, EM, HB, CB, CG-T, PF, HL-M, CBL, BV, DM-G and FS. Manuscript draft writing: DB. Manuscript draft review and validation and final approval of manuscript: All authors.

\section{FUNDING}

Roche France and GINECO. Medical writing support was provided by Jennifer Kelly, MA (Medi-Kelsey Limited, Ashbourne, United Kingdom), funded by GINECO.

\section{ACKNOWLEDGMENTS}

We thank the investigators at the 103 centers that contributed to this trial, all the women who participated in this trial, and their families. 


\section{REFERENCES}

Avastin Prescribing Information (2020). Available at: https://www.accessdata. fda.gov/drugsatfda_docs/label/2020/125085s332lbl.pdf (Accessed Mar 24, 2021).

Burger, R. A., Brady, M. F., Bookman, M. A., Fleming, G. F., Monk, B. J., Huang, H., et al. (2011). Incorporation of Bevacizumab in the Primary Treatment of Ovarian Cancer. N. Engl. J. Med. 365, 2473-2483. doi:10.1056/ NEJMoa 1104390

de la Motte Rouge, T., Ray-Coquard, I., and You, B. (2019). [Medical Treatment in Ovarian Cancers Newly Diagnosed: Article Drafted from the French Guidelines in Oncology Entitled "Initial Management of Patients with Epithelial Ovarian Cancer" Developed by FRANCOGYN, CNGOF, SFOG, GINECO-ARCAGY under the Aegis of CNGOF and Endorsed by INCa]. Gynecol. Obstet. Fertil. Senol 47, 222-237. doi:10.1016/j.gofs.2019.01.002

Hall, M., Bertelli, G., Li, L., Green, C., Chan, S., Yeoh, C. C., et al. (2020). Role of Front-Line Bevacizumab in Advanced Ovarian Cancer: the OSCAR Study. Int. J. Gynecol. Cancer 30, 213-220. doi:10.1136/ijgc-2019-000512

Institut National du Cancer (INCa) (2021). Tumeurs solides - Estimations nationales de l'incidence et de la mortalité par cancer en France métropolitaine entre 1990 et 2018. Available at: https://www.e-cancer.fr/ Expertises-et-publications/Catalogue-des-publications/Rapport-Volume-1Tumeurs-solides-Estimations-nationales-de-l-incidence-et-de-la-mortalitepar-cancer-en-France-metropolitaine-entre-1990-et-2018-juillet-2019 (Accessed Mar 24, 2021).

Komiyama, S., Kato, K., Inokuchi, Y., Takano, H., Matsumoto, T., Hongo, A., et al. (2019). Bevacizumab Combined with Platinum-Taxane Chemotherapy as First-Line Treatment for Advanced Ovarian Cancer: a Prospective Observational Study of Safety and Efficacy in Japanese Patients (JGOG3022 Trial). Int. J. Clin. Oncol. 24, 103-114. doi:10.1007/s10147018-1319-y

Marth, C., Reimer, D., and Zeimet, A. G. (2017). Front-line Therapy of Advanced Epithelial Ovarian Cancer: Standard Treatment. Ann. Oncol. 28 (Suppl. 8), viii36-viii39. doi:10.1093/annonc/mdx450

Miles, D., Bridgewater, J., Ellis, P., Harrison, M., Nathan, P., Nicolson, M., et al. (2010). Using Bevacizumab to Treat Metastatic Cancer: UK Consensus Guidelines. Br. J. Hosp. Med. (Lond) 71, 670-677. doi:10.12968/ hmed.2010.71.12.670

Musella, A., Vertechy, L., Romito, A., Marchetti, C., Giannini, A., Sciuga, V., et al. (2017). Bevacizumab in Ovarian Cancer: State of the Art and Unanswered Questions. Chemotherapy 62, 111-120. doi:10.1159/000448942

Oza, A. M., Selle, F., Davidenko, I., Korach, J., Mendiola, C., Pautier, P., et al. (2017). Efficacy and Safety of Bevacizumab-Containing Therapy in Newly Diagnosed Ovarian Cancer: ROSiA Single-Arm Phase 3B Study. Int. J. Gynecol. Cancer 27, 50-58. doi:10.1097/IGC.0000000000000836

Perren, T. J., Swart, A. M., Pfisterer, J., Ledermann, J. A., Pujade-Lauraine, E., Kristensen, G., et al. (2011). A Phase 3 Trial of Bevacizumab in Ovarian Cancer. N. Engl. J. Med. 365, 2484-2496. doi:10.1056/NEJMoa1103799

Pfisterer, J., Joly, F., Kristensen, G., Rau, J., Mahner, S., Pautier, P., et al. (2021). Optimal Treatment Duration of Bevacizumab (BEV) Combined with Carboplatin and Paclitaxel in Patients (Pts) with Primary Epithelial Ovarian (EOC), Fallopian Tube (FTC) or Peritoneal Cancer (PPC): A Multicenter Open-Label Randomized 2-arm Phase 3 ENGOT/GCIG Trial of the AGO Study Group, GINECO, and NSGO (AGO-OVAR 17/BOOST, GINECO OV118, ENGOT Ov-15, NCT01462890). J. Clin. Oncol. 39 (Suppl. 15), 5501. doi:10.1200/JCO.2021.39.15_suppl.5501
Pignata, S., Lorusso, D., Joly, F., Gallo, C., Colombo, N., Sessa, C., et al. (2021). Carboplatin-Based Doublet Plus Bevacizumab Beyond Progression Versus Carboplatin-Based Doublet Alone in Patients with Platinum-Sensitive Ovarian Cancer: A Randomised, Phase 3 Trial. Lancet Oncol. 22, 267-276. doi:10.1016/S1470-2045(20)30637-9

Plummer, C., Michael, A., Shaikh, G., Stewart, M., Buckley, L., Miles, T., et al. (2019). Expert Recommendations on the Management of Hypertension in Patients with Ovarian and Cervical Cancer Receiving Bevacizumab in the UK. Br. J. Cancer 121, 109-116. doi:10.1038/s41416-019-0481-y

Ray-Coquard, I., Pautier, P., Pignata, S., Pérol, D., González-Martín, A., Berger, R., et al. (2019). Olaparib Plus Bevacizumab as First-Line Maintenance in Ovarian Cancer. N. Engl. J. Med. 381, 2416-2428. doi:10.1056/NEJMoa1911361

Sehouli, J., Mustea, A., Oskay-Ozcelik, G., Keller, M., Richter, R., Tomé, O., et al. (2020). Safety and Effectiveness of Bevacizumab (BEV)-Containing Therapy in Patients (Pts) with Primary Ovarian Cancer (OC): Interim Analyses of the OTILIA German Non-Interventional Study. Oncol. Res. Treat. 43 (Suppl. 1), 93 (Abstract 684), 2020. Poster presented at Deutscher Krebskongress, Berlin, Germany. doi:10.1159/000506491

Conflict of Interest: AF reports honoraria and travel/accommodation/expenses from AstraZeneca, Clovis, and GSK, and non-remunerated activities for MSD and Roche. PT reports honoraria from MSD, Tesaro/GSK, and Pfizer, and advisory/ consultancy roles for MSD and Tesaro/GSK. JA reports honoraria from GSK AstraZeneca, MSD, PharmaMar, and Eisai; travel/accommodation/expenses from Novartis, Janssen, and GSK; and research grant/funding to his institution from Janssen and MSD. CG-T reports honoraria from and advisory/consultancy roles for Pfizer; speaker bureau/expert testimony for AstraZeneca and MSD; travel/ accommodation/expenses from MSD, Pfizer, and Mylan; and research grant/ funding to her institution from Roche. PF reports honoraria from and advisory/consultancy roles for Novartis, AstraZeneca, GSK, and Clovis, and travel/accommodation/expenses from Novartis, AstraZeneca, and GSK. CBL reports honoraria (paid to his institution) from Roche, Novartis, Isofol, Array Biopharma, AstraZeneca, BMS, and Celgene. FS reports honoraria from Roche, AstraZeneca, Tesaro, Clovis, MSD, PharmaMar, and GlaxoSmithKline; advisory/ consultancy role for Roche; speaker bureau/expert testimony for Roche, AstraZeneca, Tesaro, and GlaxoSmithKline; and travel/accommodation/ expenses from Roche, AstraZeneca, Tesaro, MSD, and PharmaMar.

The remaining authors declare that the research was conducted in the absence of any commercial or financial relationships that could be construed as a potential conflict of interest.

Publisher's Note: All claims expressed in this article are solely those of the authors and do not necessarily represent those of their affiliated organizations, or those of the publisher, the editors and the reviewers. Any product that may be evaluated in this article, or claim that may be made by its manufacturer, is not guaranteed or endorsed by the publisher.

Copyright (C) 2021 Berton, Floquet, Lescaut, Baron, Kaminsky, Toussaint, Largillier, Savoye, Alexandre, Delbaldo, Malaurie, Barletta, Bosacki, Garnier-Tixidre, Follana, Laharie-Mineur, Briac Levache, Valenza, Dechartres, Mollon-Grange and Selle. This is an open-access article distributed under the terms of the Creative Commons Attribution License (CC BY). The use, distribution or reproduction in other forums is permitted, provided the original author(s) and the copyright owner(s) are credited and that the original publication in this journal is cited, in accordance with accepted academic practice. No use, distribution or reproduction is permitted which does not comply with these terms. 